\title{
A Note on the Boson-Fermion Correspondence and Infinite Dimensional Groups
}

\author{
A. L. Carey ${ }^{1}$ and C. A. Hurst ${ }^{2}$ \\ 1 Department of Pure Mathematics and \\ 2 Department of Mathematical Physics, University of Adelaide, G.P.O. Boc 498, Adelaide, \\ South Australia 5001
}

\begin{abstract}
We show how to construct irreducible projective representations of the infinite dimensional Lie group $\operatorname{Map}\left(S^{1}, \mathbb{T}\right)$, by embedding it into the group of Bogoliubov automorphisms of the CAR. Using techniques of G. Segal for extending certain representations of Map $\left(S^{1}, S U(2)\right)$ we show that our representations extend to give representations of a certain infinite dimensional superalgebra. We relate our work to the well known boson-fermion correspondence which exists in $1+1$ dimensions.
\end{abstract}

\section{Introduction}

Recently Frenkel and Kac [1] constructed representations of certain infinite dimensional Lie algebras. While this note was in preparation Frenkel related their work to current algebras and the boson-fermion correspondence [2]. Now in $1+1$ dimensional field theory one can construct representations of current algebras using automorphisms of the CAR algebra for the $1+1$ dimensional Dirac field [3]. We became interested in the connection between [1 and 3] as a result of the work of Graeme Segal [4] in which he constructs projective representations of some infinite dimensional Lie groups of which the Frenkel-Kac representations are the Lie algebra versions.

The resemblance between [ 3 and 4$]$ is intriguing although the representations determined by us have more to do with Lie superalgebras than Kac-Moody algebras. It would be interesting to explore the superalgebra connection further. However we content ourselves in this note with the problem of making explicit the connection between [3 and 4], and hence with [1].

We adopt a simplified model of fermions, namely we construct the CAR algebra over $L^{2}\left(S^{1}, \mathbb{C}\right)$. In field theoretic terms this amounts to taking the massless Dirac equation in $1+1$ dimensions in "light cone coordinates" so that the 2 components of the field can be separately dealt with and then imposing a periodic box cutoff. We do this simply because all the calculations can then be made explicit. 
The group $\operatorname{Map}\left(S^{1}, \mathbb{T}\right)$ of smooth maps from $S^{1}$ to the circle group acts on $L^{2}\left(S^{1}, \mathbb{C}\right)$ as unitary multiplication operators. As such it imbeds in the group of Bogoliubov transformations of the CAR algebra over $L^{2}\left(S^{1}, \mathbb{C}\right)$. Representations of this group of Bogoliubov transormations then determine (projective) representations of $\operatorname{Map}\left(S^{1}, \mathbb{T}\right)$. In Sect. 2 we make this explicit for a particular case and determine the 2-cocycle on $\operatorname{Map}\left(S^{1}, \mathbb{T}\right)$ which so arises. It turns out to be a cocycle of winding number one in Segal's terminology [4]. In contrast [1, 2, 4] are concerned with cocycles with winding number two. Our case is of interest because in principle representations corresponding to a cocycle of winding number $n$ can be obtained by decomposing a tensor product of $n$ copies of a winding number one cocycle representation.

In [1] and [4] an "extension theorem" is proved which shows that certain projective representations of $\operatorname{Map}\left(S^{1}, \mathbb{T}\right)$ extend canonically to $\operatorname{Map}\left(S^{1}, \operatorname{SU}(2)\right)$. For our case this result amounts to a special version of the construction employed by Dell-Antonio, Frishman and Zwanziger [5] (in connection with the massless Thirring model). There they showed how to take certain boson fields (in fact currents) and construct fermion fields as functions of them. This is an example of the boson-fermion correspondence which exists in $1+1$ dimensions (and originally exploited by Coleman [6]).

Thus our second result is an explicit operator version (for free fields) of this bosen-fermion correspondence. To some extent this settles doubts which have arisen as to the validity of the boson-fermion correspondence $[7,8]$.

We conclude with some miscellaneous remarks about the Lie group representations obtained in this note and about "models which predict their own superselection sectors."

A different approach to these questions has been taken by Heindenreich et al. [9] and Frenkel [2] using Lie algebra methods. The former paper obtains similar results while ignoring points of rigor.

Frenkel [2] on the other hand has examined the boson-fermion correspondence for a different current algebra leading to results analogous to ours for "QED-like" models in $1+1$ dimensions. It would be useful to understand in more detail the relationship between our approach and [2], although superficially his construction is a "tensor product" version of ours.

\section{Automorphisms of CAR}

We begin with a discussion of the notion of index of a Bogoliubov transformation developed in [9]. We let $\mathscr{H}$ be a complex Hilbert space and $\mathscr{A}$ the $C^{*}$-algebra of the CAR over $\mathscr{H}$ generated by $\left\{a(h), a^{*}(h) \mid h \in \mathscr{H}\right\}$ with

$$
\begin{gathered}
a(h) a(g)+a(g) a(h)=0, \\
a^{*}(h) a(g)+a(g) a^{*}(h)=\langle g, h\rangle_{\mathscr{H}} .
\end{gathered}
$$

If $P_{+}$and $P_{-}$are orthogonal projections on $\mathscr{H}$ with $P_{+}+P_{-}=I$ then an irreducible representation $\pi_{P_{-}}$of $\mathscr{A}$ is defined by the state $\omega_{P_{-}}$:

$$
\omega_{P_{-}}\left(a^{*}\left(h_{1}\right) a^{*}\left(h_{2}\right) \ldots a^{*}\left(h_{n}\right) a\left(g_{m}\right) \ldots a\left(g_{1}\right)\right)=\delta_{n m} \operatorname{det}\left\langle g_{i}, P_{-} h_{j}\right\rangle_{\mathscr{H}} .
$$


We let $\mathscr{U}$ denote the group of unitary operators $U$ on $\mathscr{H}$ such that $P_{+} U P_{-}$and $P_{-} U P_{+}$are Hilbert-Schmidt. Then $\mathscr{U}$ is the group of operators for which the automorphism of $\mathscr{A}$ defined by $a(h) \rightarrow a(U h)$ is implemented in $\pi_{P_{-}}$in the sense that there exists a unitary operator $\Gamma(U)$ in the Hilbert space of $\pi_{P_{-}}$such that

$$
\Gamma(U) \pi_{P_{-}}(a(h)) \Gamma(U)^{-1}=\pi_{P_{-}}(a(U h)) .
$$

From the relations $U U^{*}=I$ and $U^{*} U=I$ we have

$$
P_{+} U P_{+} U^{*} P_{+}+P_{+} U P_{-} U^{*} P_{+}=P_{+}=P_{+} U^{*} P_{+} U P_{+}+P_{+} U^{*} P_{-} U P_{+},
$$

so that $P_{+} U P_{+}$is invertible modulo the ideal of compact operators on $P_{+} \mathscr{H}$ and so is Fredholm. Moreover when $P_{+}$and $P_{-}$both have infinite dimensional range, the map $i: U \rightarrow$ Fredholm index of $P_{+} U P_{+}$has the properties

(i) $i: \mathscr{U} \rightarrow \mathbb{Z}$ is a homomorphism;

(ii) $\mathrm{ker} i$ is the connected component of the identity of $\mathscr{U}$ in the topology defined below.

We can consider two topologies on $\mathscr{U}$ determined by saying that a set $\left\{\mathscr{U}_{\alpha}\right\}$ in $\mathscr{U}$ converges to $U$ if and only if

$$
P_{+} U_{\alpha} P_{-}+P_{-} U_{\alpha} P_{+} \rightarrow P_{+} U P_{-}+P_{-} U P_{+},
$$

in Hilbert-Schmidt norm and

$$
P_{+} U_{\alpha} P_{+}+P_{-} U_{\alpha} P_{-} \rightarrow P_{+} U P_{+}+P_{-} U P_{-},
$$

in either the uniform or the strong operator topology. The former topology is rather more natural from the viewpoint of the Fredholm theory used in [10], while the latter is more natural if one considers the question of continuity of $U \rightarrow \Gamma(U)$ as a (projective) representation (see [11]). In either topology the connected components are the same [11].

We now specialize to the case where $\mathscr{H}=L^{2}\left(S^{1}, \mathbb{C}\right)$ with $S^{1}$ the unit circle and $P_{+}$and $P_{-}$chosen to project onto the positive (plus zero) and negative Fourier components respectively. So if

then

$$
h(s)=\frac{1}{\sqrt{2 \pi}} \sum_{n} h_{n} e^{i n s}, \quad s \in[0,2 \pi),
$$

$$
\left(P_{-} h\right)(s)=\frac{1}{\sqrt{2 \pi}} \sum_{n<0} h_{n} e^{i n s} .
$$

Of course $P_{ \pm} \mathscr{H}=\mathscr{H}_{ \pm}$are the usual Hardy spaces associated with the unit disc consisting of functions analytic inside (outside) the disc and $L^{2}$ on the boundary $S^{1}$.

A particular class of automorphisms of the CAR over $\mathscr{H}$ was considered in [3]. Let $\mathscr{M}=\operatorname{Map}\left(S^{1}, \mathbb{T}\right)$ and observe that $\mathscr{M}$ acts on $L^{2}\left(S^{1}, \mathbb{C}\right)$ by multiplication:

$$
\left(U_{\phi} g\right)(s)=\phi(s) g(s), \quad \phi \in \mathscr{M}, \quad g \in L^{2}\left(S^{1}, \mathbb{C}\right) .
$$

That $\left\{U_{\phi} \mid \phi \in \mathscr{M}\right\}$ forms a subgroup of $\mathscr{U}$ follows using

$$
\begin{aligned}
\operatorname{trace}\left(P_{-} U_{\phi}^{*} P_{+} U_{\phi} P_{-}\right) & =\frac{1}{2 \pi} \sum_{k=1}^{\infty} k \phi_{k}^{*} \phi_{k} \\
& <\infty
\end{aligned}
$$


for $\phi$ smooth and

$$
\phi(s)=\frac{1}{\sqrt{2 \pi}} \sum_{k} \phi_{k} e^{i k s} .
$$

For this simple case the standard theory of Toeplitz operators [12] gives $i\left(U_{\phi}\right)=-w(\phi)$, where $w(\phi)$ is the winding number of $\phi$. By forming the representation $\pi_{P_{-}}$of the CAR algebra over $L^{2}\left(S^{1}, \mathbb{C}\right)$ we obtain a projective representation $\phi \rightarrow \Gamma\left(U_{\phi}\right)$ of $\mathscr{M}$. The connected component of the identity of $\mathscr{M}$, say $\mathscr{M}_{0}$, consists of those $\phi$ with winding number zero. As in [4], every $\phi \in \mathscr{M}$ can be written $\phi=e^{i f}$ with $f: \mathbb{R} \rightarrow \mathbb{R}$ satisfying

$$
f(s+2 \pi)-f(s)=2 \pi n, \quad n \in \mathbb{Z}
$$

independently of $s \in S^{1}$.

Our aim in the rest of this section is to describe in some detail the projective representation $\phi \rightarrow \Gamma\left(U_{\phi}\right)$ of $\mathbb{M}$. Although for each $\phi \in \mathscr{M}, \Gamma\left(U_{\phi}\right)$ is determined only up to a phase factor, given any pair $\phi_{1}, \phi_{2} \in \mathscr{M}$ with $\phi_{j}=\exp i f_{j}(j=1,2)$, there is a real number $\tilde{\sigma}\left(f_{1}, f_{2}\right)$ such that

$$
\Gamma\left(U_{\phi_{1}}\right) \Gamma\left(U_{\phi_{2}}\right)=\exp -i \tilde{\sigma}\left(\mathrm{f}_{1}, f_{2}\right) \Gamma\left(U_{\phi_{2}}\right) \Gamma\left(U_{\phi_{1}}\right),
$$

and which clearly does not depend on the choice of phase for $\Gamma\left(U_{\phi_{j}}\right)$.

On the other hand if we make a choice of phase for each $\Gamma\left(U_{\phi}\right)$, then we determine a 2-cocycle on $\mathscr{M}$ from the relation

with

$$
\Gamma\left(U_{\phi_{1}}\right) \Gamma\left(U_{\phi_{2}}\right)=\exp -i \sigma\left(f_{1}, f_{2}\right) \cdot \Gamma\left(U_{\phi_{1} \phi_{2}}\right)
$$

$$
\tilde{\sigma}\left(f_{1}, f_{2}\right)=\sigma\left(f_{1}, f_{2}\right)-\sigma\left(f_{2}, f_{1}\right) .
$$

Now from [13] or [15] one knows that the 2-cocycle identity for the cocycle

$$
\phi_{1}, \phi_{2} \rightarrow \exp -i \sigma\left(f_{1}, f_{2}\right)
$$

implies that the map $\phi_{1}, \phi_{2} \rightarrow \exp -i \tilde{\sigma}\left(f_{1}, f_{2}\right)$ is a character in each variable separately and furthermore $[13,14]$ that it determines the cohomology class of $\sigma$ completely. Our first task then will be to calculate $\tilde{\sigma}$.

For each $\phi=e^{i f} \in \mathscr{M}_{0}$ we have a one parameter group $r \rightarrow e^{i r f}=\phi_{r}$ in $\mathscr{M}_{0}(r \in \mathbb{R})$. From [16] we know that we may choose the phase of $\Gamma\left(U_{\phi_{r}}\right)$ so that $r \rightarrow \Gamma\left(U_{\phi_{r}}\right)$ is a strongly continuous one parameter group and hence $\Gamma\left(U_{\phi_{r}}\right)=\operatorname{expir} J(f)$ with $J(f)$ (unbounded) self adjoint. One also has [16] the fact that vectors of the form

$$
\pi_{P_{-}}\left(a^{*}\left(h_{1}\right) \ldots a^{*}\left(h_{n}\right) a\left(g_{m}\right) \ldots a\left(g_{1}\right)\right) \Omega
$$

are in the domain of $J(f)$ and hence the phase of $\Gamma\left(U_{\phi_{r}}\right)$ is completely specified by requiring that $\langle\Omega, J(f) \Omega\rangle=0$. We now record

Lemma 2.1 [16]. For $\phi_{j}=e^{i f_{j}} \in \mathscr{M}_{0}, j=1,2$,

where

$$
\tilde{\sigma}\left(f_{1}, f_{2}\right)=2 \operatorname{Im} \operatorname{tr}\left(P_{-} f_{1} P_{+} f_{2} P_{-}\right)=\frac{1}{2 \pi \mathrm{i}} \sum_{k} k\left(f_{1}\right)_{k}^{*}\left(f_{2}\right)_{k}
$$

$$
f_{j}(s)=\frac{1}{\sqrt{2 \pi}} \sum_{k}\left(f_{j}\right)_{k} e^{i k s}
$$


We omit the proof as it follows directly from the results of [16]. The formula (2.2) for $\tilde{\sigma}\left(f_{1}, f_{2}\right)$ may be rewritten as

$$
\tilde{\sigma}\left(f_{1}, f_{2}\right)=\frac{1}{4 \pi} \int_{0}^{2 \pi}\left[\frac{d f_{1}(s)}{d s} f_{2}(s)-f_{1}(s) \frac{d f_{2}(s)}{d s}\right] d s .
$$

Lemma 2.2. Let $V g(s)=e^{i s} g(s)$ and $\phi=e^{i f} \in \mathscr{M}_{0}$. Then

$$
\left\langle\Omega, \Gamma(V)^{-1} \Gamma\left(U_{\phi}\right) \Gamma(V) \Omega\right\rangle=\exp \left(\frac{i}{\sqrt{2 \pi}} f_{0}\right)\left\langle\Omega, \Gamma\left(U_{\phi}\right) \Omega\right\rangle,
$$

where the phase of $\Gamma\left(U_{\phi}\right)$ is chosen as in the paragraph preceding Lemma 2.1 and $f_{0}=\frac{1}{\sqrt{2 \pi}} \int_{0}^{2 \pi} f(s) d s$.

Proof. Notice that the relation we have to prove does not depend on a choice of phase for $\Gamma(V)$, so we may make for the moment some arbitary choice. Then the vector $\Gamma(V) \Omega$ defines the state $\omega_{V P_{-} V_{-1}}$ on $\mathscr{A}$ corresponding to the projection $P_{-}^{\prime}=V P_{-} V^{-1}$. On the other hand, with $e_{0}(s)=\frac{1}{\sqrt{2 \pi}}, s \in S^{1}$, the vector $\pi_{P_{-}}\left(a^{*}\left(e_{0}\right)\right) \Omega$ satisfies

$$
\pi_{P_{-}}\left[a^{*}\left(P_{-}^{\prime} g\right) a^{*}\left(e_{0}\right)\right] \Omega=0=\pi_{P_{-}}\left[a\left(\left(1-P_{-}^{\prime}\right) g\right) a^{*}\left(e_{0}\right)\right] \Omega
$$

for all $g \in L^{2}\left(S^{1}, \mathbb{C}\right)$ so that

$$
\left\langle\pi_{P_{-}}\left(a^{*}\left(e_{0}\right)\right) \Omega, \pi_{P_{-}}\left(a^{*}(h) a(g)\right) \pi_{P_{-}}\left(a^{*}\left(e_{0}\right)\right) \Omega\right\rangle=\left\langle g, P_{-}^{\prime} h\right\rangle_{L^{2}} .
$$

Hence one may conclude that the vector $\pi_{P_{-}}\left(a^{*}\left(e_{0}\right)\right) \Omega$ defines the same state on $\mathscr{A}$ as $\Gamma(V) \Omega$. But $\pi_{P_{-}}$is irreducible and so these two vectors differ by a phase (see also Ruijsenaars [17] for this fact). But then

$$
\left\langle\Omega, \Gamma(V)^{-1} \Gamma\left(U_{\phi}\right) \Gamma(V) \Omega\right\rangle=\left\langle e_{0}, f \cdot e_{0}\right\rangle_{L^{2}},
$$

from which the result follows.

Now write $V=e^{i v}$ and note that $\tilde{\sigma}(n v, f)=\frac{n}{\sqrt{2 \pi}} f_{0}$ by Lemma 2.2, so that for $\phi_{1}$, $\phi_{2}$ having winding numbers $n_{1}, n_{2}$ respectively we have $\exp i\left(f_{1}-n_{1} v\right)$ and $\exp i\left(f_{2}-n_{2} v\right)$ in $\mathscr{M}_{0}$. Using the character property of $e^{i \tilde{\sigma}}$ we have

$$
\begin{aligned}
e^{i \tilde{\sigma}\left(f_{1}, f_{2}\right)}= & \exp i\left[\tilde{\sigma}\left(f_{1}-n_{1} v, f_{2}-n_{2} v\right)+\tilde{\sigma}\left(n_{1} v, f_{2}-n_{2} v\right)+\tilde{\sigma}\left(n_{1} v, n_{2} v\right)\right. \\
& \left.+\tilde{\sigma}\left(f_{1}-n_{1} v, n_{2} v\right)\right] .
\end{aligned}
$$

By (2.2) and Lemma 2.2, the only term on the right-hand side which we have not determined is $e^{i \tilde{\sigma}\left(n_{1} v, n_{2} v\right)}$, and this is easily seen to be identically one. We now find that

$$
\begin{aligned}
\tilde{\sigma}\left(f_{1}, f_{2}\right)= & \frac{1}{4 \pi} \int_{0}^{2 \pi}\left[\frac{d f_{1}(s)}{d s} f_{2}(s)-f_{1}(s) \frac{d f_{2}(s)}{d s}\right] d s \\
& +\frac{1}{4 \pi}\left[f_{1}(2 \pi) f_{2}(0)-f_{1}(0) f_{2}(2 \pi)\right] .
\end{aligned}
$$


We now develop some consequences of the preceding calculations. Denote by $\mathscr{V}$ the space of smooth functions $f$ from $S^{1}$ to $\mathbb{R}$ such that $\int_{0}^{2 \pi} f(s) d s=0$. Notice firstly that (2.1) implies the relation, for $f, f^{\prime} \in \mathscr{V}$ :

$$
\left[J(f), J\left(f^{\prime}\right)\right]_{-} \subseteq i \tilde{\sigma}\left(f, f^{\prime}\right)=\frac{1}{2 \pi} \sum_{k} k f_{k}^{*} f_{k}^{\prime} .
$$

Now observe that $\tilde{\sigma}$ is a non-degenerate skew-symmetric form on $\mathscr{V}$ which is the imaginary part of the inner product:

$$
\left\langle f, f^{\prime}\right\rangle=\frac{1}{\pi} \sum_{k>0} k f_{k}^{*} f_{k}^{\prime} .
$$

If $\overline{\mathscr{V}}$ denotes the completion of $\mathscr{V}$ in the resulting norm then $\mathscr{V}$ becomes a complex Hilbert space where multiplication by $\sqrt{-1}$ is defined by the complex structure $C$,

Now define

$$
(C f)_{k}=i \varepsilon(k) f_{k}, \varepsilon(k)= \begin{cases}+1 & k>0 \\ -1 & k<0\end{cases}
$$

$$
\beta(f)=\frac{1}{2}(J(\mathrm{f})+i J(C f)), \beta(f)^{*}=\frac{1}{2}(J(f)-i J(C f)) .
$$

The general results of [16] when applied to this situation give the relation

$$
\left\langle\Omega, J\left(f_{1}\right) J\left(f_{2}\right) \Omega\right\rangle=\frac{1}{2}\left\langle f_{1}, f_{2}\right\rangle \text {. }
$$

One also has

$$
\left[\beta\left(f_{1}\right), \beta\left(f_{2}\right)^{*}\right]_{-}=\frac{1}{2}\left\langle f_{1}, f_{2}\right\rangle
$$

and from (2.5)

$$
\|\beta(f) \Omega\|^{2}=0,
$$

and hence $\beta(f) \Omega=0$ for all $f \in \mathscr{V}$. Thus $\beta(f)$ and $\beta(f)^{*}$ are annihilation and creation operators while (2.6) to (2.7) imply by standard arguments that

$$
\langle\Omega, \operatorname{expiJ}(f) \Omega\rangle=\exp -\frac{1}{4}\langle f, f\rangle .
$$

We conclude then that the cyclic representation of $\mathscr{M}_{0}$ generated from $\Omega$ by the $\Gamma\left(U_{\phi}\right), \phi \in \mathscr{M}_{0}$, is just the Fock representation of the canonical commutation relations (2.3). That is $f \rightarrow J(f), f \in \mathscr{V}$ is a "free bose field."

Theorem 2.3. The projective representation $\phi \rightarrow \Gamma\left(U_{\phi}\right)$ of $\mathscr{M}$ is irreducible. On restriction to $\mathscr{M}_{0}$ the Hilbert space decomposes as a direct sum $\bigoplus_{k \in \mathbb{Z}} \mathscr{H}_{k}$, where $\mathscr{H}_{k}$ all carry the Fock representation of the CCR (2.3) and the scalars $\gamma$ in $\mathscr{M}_{0}$ act on $\mathscr{H}_{k}$ by multiplication by $\gamma^{k}$.

Proof. We could appeal to Uhlenbrock [18] for this result. Alternatively the proof of the main result of [3] can be adapted to this situation. We sketch how this goes. Firstly define vacua $\Omega_{k}$ for the boson field $f \rightarrow J(f), f \in \bar{V}$ by $\Omega_{k}=\Gamma(V)^{k} \Omega$. Then $\mathscr{H}_{k}$ is defined to be the subspace of the Hilbert space $\mathscr{F}$, say, of $\pi_{P_{-}}$, generated from 
$\Omega_{k}$ by the action of $\left\{\Gamma\left(U_{\phi}\right) \mid \phi \in \mathscr{M}_{0}\right\}$. It is straightforward to verify that $\mathscr{F}$ decomposes into a direct sum, indexed by $\mathbb{Z}$, of the representations of the constant subgroup of $\mathscr{M}_{0}$. [This is because the generator of the one parameter group $r \rightarrow \Gamma\left(e^{i r}\right), r \in \mathbb{R}$, is the usual charage operator for the representation $\pi_{P_{-}}$of $\mathscr{A}$.] The only problem then is to check that the space $\mathscr{H}_{k}$ exactly coincides with the space on which the constants $\gamma$ act by $\gamma^{k}$ since each $\mathscr{H}_{k}$ carries the Fock representation of the CCR (2.3). Now the $\Gamma(V)^{k}$ intertwine the different spaces so that it is sufficient to verify that $\mathscr{H}_{0}$ coincides with the space on which the constants act trivially. In [3] we developed an argument, using this "Hardy space" picture of the fermion and boson fields, which showed precisely this fact. This concludes the proof.

Let us now complete this aspect of the discussion of the projective representation of $\mathscr{M}$ by giving a phase choice which leads to the 2-cocycle on $\mathscr{M}$ introduced in [4]. This cocycle is given by

$$
\sigma\left(f_{1}, f_{2}\right)=\frac{1}{4 \pi} \int_{0}^{2 \pi} \frac{d f_{1}(s)}{d s} f_{2}(s) d s+\frac{1}{4 \pi}\left(f_{1}(2 \pi)-f_{1}(0)\right) f_{2}(0) .
$$

An appropriate phase choice leading to (2.9) is given by choosing the phase of $\Gamma\left(U_{\phi}\right)$, for $\phi \in \mathscr{M}_{0}$ as in the discussion preceding Lemma 2.1, choosing that for $\Gamma(V)$ so that $\Gamma(V) \Omega=\pi_{P_{-}}\left(a^{*}\left(e_{0}\right)\right) \Omega$, and then defining

$$
\begin{aligned}
& \Gamma\left(V^{n}\right)=\Gamma(V)^{n} \operatorname{expin}(n-1) \sigma(v, v) / 2, \\
& \Gamma\left(U_{\phi}\right)=\Gamma\left(U_{\phi} V^{-n}\right) \Gamma\left(V^{n}\right) \exp i \sigma(f-n v, n v),
\end{aligned}
$$

where $n=$ winding number of $\phi \in \mathscr{M}$. It is then straightforward to check that the relation

$$
\Gamma\left(U_{\phi_{1}}\right) \Gamma\left(U_{\phi_{2}}\right)=e^{-i \sigma\left(f_{1}, f_{2}\right)} \Gamma\left(U_{\phi_{1} \phi_{2}}\right)
$$

holds for all $\varphi_{1}, \varphi_{2}$ in $\mathscr{M}$.

We turn now to a result which will be important in the next section. Following [4] one may define an action of a double cover of the circle group $\mathbb{T}$ on the central extension of $\mathscr{M}$ determined by $\sigma$ via

$$
r_{\alpha}(z, \phi)=\left(z \exp \left[-\frac{i}{2} n(f(\alpha)-f(0))\right], \phi_{\alpha}\right),
$$

where $z \in \mathbb{T}, \alpha \in[0,2 \pi], \phi=e^{i f} \in \mathscr{M}, n$ is the winding number of $\phi$ and $\phi_{\alpha}\left(\alpha^{1}\right)=\phi\left(\alpha+\alpha^{1}\right)$. This action is automorphic and furthermore we have

Lemma 2.4. The action (2.10) is implemented on $\mathscr{F}$ by the operator $R(\alpha)$, where

$$
R(\alpha) \Gamma\left(V^{n}\right) \beta\left(f_{1}\right)^{*} \ldots \beta\left(f_{m}\right)^{*} \Omega=e^{i n^{2} \alpha / 2} \Gamma\left(V^{n}\right) \beta\left(f_{1_{\alpha}}\right)^{*} \ldots \beta\left(f_{m_{\alpha}}\right)^{*} \Omega, \text { with } f_{j} \in \overline{\mathscr{V}},
$$
$f_{j_{\alpha}}\left(\alpha^{1}\right)=f_{j}\left(\alpha+\alpha^{1}\right), j=1, \ldots, m$.

Proof. The action of $R(\alpha)$ on $\mathscr{H}_{0}$ is determined by the fact that the map $f \rightarrow f_{\alpha}$ is unitary on $\bar{V}$ and so is implemented in the Fock representation on $\mathscr{H}_{0}$. Consequently it is sufficient to determine the action of $R(\alpha)$ on the vectors $\Gamma\left(V^{n}\right) \Omega$. 
But this is forced by requiring $R(\alpha)$ to implement (2.10):

$$
\begin{aligned}
R(\alpha) \Gamma\left(V^{n}\right) \Omega & =R(\alpha) \Gamma\left(V^{n}\right) R(\alpha)^{-1} \Omega \\
& =e^{-i n^{2} \alpha / 2} \Gamma\left(e^{i n \alpha} V^{n}\right) \Omega \\
& =e^{i n^{2} \alpha / 2} \Gamma\left(V^{n}\right) \Omega .
\end{aligned}
$$

We conclude this section by introducing some notation.

As we have a representation $\pi_{P_{-}}$of the CAR algebra over $L^{2}\left(S^{1}, \mathbb{C}\right)$, we may define "fermion fields" by

$$
\psi(h)=\pi_{P_{-}}(a(h)), \quad h \in L^{2}\left(S^{1}, C\right) .
$$

Annihilation and creation operators of "momentum" $k \in \mathbb{Z}$ are defined by

$$
\begin{array}{ll}
a_{k}=\psi\left(e_{k}\right), & k \geqq 0 \text { ("particle" annihilator), } \\
b_{k}^{*}=\psi\left(e_{-k}\right), & k>0 \text { ("antiparticle" creator), }
\end{array}
$$

where $e_{k}(s)=\frac{1}{\sqrt{2 \pi}} e^{i k s} s \in S^{1}$.

Remark 2.5. We may make contact with the usual field theory formalism by introducing the quadratic form

$$
\psi(s)=\frac{1}{\sqrt{2 \pi}} \sum_{-\infty}^{\infty} e^{i l s}\left(\theta\left(l+\frac{1}{2}\right) a_{l}+\theta\left(-l-\frac{1}{2}\right) b_{-l}^{*}\right), s \in S^{1}, \text { where } \theta(x)=\left\{\begin{array}{ll}
1 & x \geqq 0 \\
0 & x<0
\end{array} .\right.
$$

As in [3] we can identify $J(f), f \in \mathscr{V}$, with the Wick ordered product:

$$
\begin{aligned}
J(f)= & \int: \psi(s)^{*} \psi(s): f(s) d s \\
= & \frac{1}{\sqrt{2 \pi}} \sum_{k l} f_{k}\left[\theta\left(k+l+\frac{1}{2}\right) \theta\left(l+\frac{1}{2}\right) a_{k+l}^{*} a_{l}+\theta\left(l+\frac{1}{2}\right) \theta\left(-k-l-\frac{1}{2}\right) b_{-k-l} a_{l}\right. \\
& \left.+\theta\left(k+l+\frac{1}{2}\right) \theta\left(-l-\frac{1}{2}\right) a_{k+l}^{*} b_{-l}^{*}-\theta\left(-k-l-\frac{1}{2}\right) \theta\left(-l-\frac{1}{2}\right) b_{-l}^{*} b_{-k-l}\right] .
\end{aligned}
$$

This is not necessary for the ensuing discussion but is useful for making contact with results in the physics literature.

\section{The Boson-Fermion Correspondence}

Physically, the basic idea of the boson-fermion correspondence in $1+1$ dimensions (cf. Coleman [6]) is that, in certain models, one can write down formal functions of boson fields having the vacuum expectation values and statistics of fermion fields and vice versa. The fact that in $1+1$ dimensions massless fermion currents are boson fields is an example, and of course the analysis of the previous sections is just another aspect of this. The problem of rigorously defining operator valued distributions having the basic properties of fermion fields by taking functions of boson fields is rather more delicate however. In this section we observe that the construction employed by Segal [4] is another case of that used by Dell-Antonio, Frishman and Zwanziger in the massless Thirring model. 
Mathematically speaking, what was observed in the previous section is that by embedding $\operatorname{Map}\left(S^{1}, \mathbb{T}\right)$ into the group of automorphisms of $\mathscr{A}$ we obtained an irreducible projective representation of $\operatorname{Map}\left(S^{1}, \mathbb{T}\right)$ acting on the Hilbert space of the representation $\pi_{P_{-}}$of $\mathscr{A}$. It is natural then to ask if the procedure can be reversed, namely is there a simple way of defining the elements of $\mathscr{A}$ in terms of the operators representing $\operatorname{Map}\left(S^{1}, \mathbb{T}\right)$.

We begin as in [4] with an approximate step function

$$
\tilde{f}_{\alpha, \lambda}: \mathbb{R} \rightarrow \mathbb{R}, \quad \alpha \in S^{1}, \quad 0 \leqq \lambda<1,
$$

defined as a sum $q_{-\alpha}+f_{\alpha, \lambda}^{+}+f_{\alpha, \lambda}^{-}$by

$$
\begin{gathered}
q_{-\alpha}(\alpha)=\alpha^{\prime}-\alpha, \\
f_{\alpha, \lambda}^{+}(\alpha)=i \ln \left(1-\lambda e^{i\left(\alpha^{\prime}-\alpha\right)}\right), \\
f_{\alpha, \lambda}^{-}(\alpha)=-i \ln \left(1-\lambda e^{-i\left(\alpha^{\prime}-\alpha\right)}\right) .
\end{gathered}
$$

As $\lambda \rightarrow 1, \tilde{f}_{\alpha, \lambda} \rightarrow 2 \pi \theta_{\alpha}$, where $\theta_{\alpha}\left(\alpha^{\prime}\right)= \begin{cases}1 & \alpha^{\prime}>\alpha \\ 1 / 2 & \alpha^{\prime}=\alpha . \text { Our representation of } \mathscr{M} \text { satisfies } \\ 0 & \alpha^{\prime}<\alpha\end{cases}$ (see Lemma 2.4) all the requirements of Proposition 4.1 of [4] (except that the cocycle is different) so that the existence of the following limits may be established in the same way. For simplicity of notation we will write in this section $\Gamma\left(U_{\phi}\right)$ as $\Gamma(\phi)$ for $\phi \in \mathscr{M}$. Let $\gamma_{\alpha, \lambda}=\exp i \tilde{f}_{\alpha, \lambda}$ and $f_{\alpha, \lambda}=\widetilde{f}_{\alpha, \lambda}-q_{-\alpha}$, then

$$
\begin{aligned}
\Gamma\left(\gamma_{\alpha, \lambda}\right)= & \exp \frac{i}{2}\left(f_{\alpha, \lambda}^{+}(0)+f_{\alpha, \lambda}^{-}(0)\right)\left(1-\lambda^{2}\right)^{1 / 2} \Gamma\left(e^{i q_{-\alpha}}\right) \\
& \cdot \exp i \beta\left(f_{\alpha, \lambda}\right)^{*} \exp i \beta\left(f_{\alpha, \lambda}\right) .
\end{aligned}
$$

[To see this, use the definition (2.9) of $\sigma$ ]

The limit

$$
B_{\alpha}=e^{i \alpha} \lim _{\lambda \rightarrow 1} \Gamma\left(e^{i q-\alpha}\right) \exp i \beta\left(f_{\alpha, \lambda}\right)^{*} \exp i \beta\left(f_{\alpha, \lambda}\right),
$$

exists just as in [4].

We sketch the arguments for the reader's convenience. With $\tilde{e}_{n}=e_{n}+e_{-n}$, let

$$
\beta_{n}^{*}=\beta\left(\tilde{e_{n}}\right)^{*}, \quad \text { and } \quad \beta_{n}=\beta\left(\tilde{e}_{n}\right), \quad n>0 .
$$

Then

$$
\beta\left(f_{\alpha, \lambda}\right)^{*}=-i \sum_{n>0} \frac{\lambda^{n}}{n} e^{-i n \alpha} \sqrt{2 \pi} \beta_{n}^{*},
$$

with a similar expression for $\beta\left(f_{\alpha, \lambda}\right)$. By expanding the exponentials in terms of $\beta_{n}^{*}$ and $\beta_{n}$, one obtains

$$
\begin{aligned}
& \exp i \beta\left(f_{\alpha, \lambda}\right)^{*}=\sum_{n} \lambda^{n} e^{-i n \alpha} C_{n}, \\
& \exp i \beta\left(f_{\alpha, \lambda}\right)=\sum_{n} \lambda^{n} e^{i n \alpha} D_{n},
\end{aligned}
$$

where $C_{n}$ maps the eigenspace, of the generator of the rotation group of $S^{1}$, in $\mathscr{F}$, corresponding to eigenvalue $q$, say $\mathscr{F}_{q}$, into $\mathscr{F}_{q+n}$ and $D_{n}$ takes $\mathscr{F}_{q}$ to $\mathscr{F}_{q-n}$ with 
$\mathscr{F}_{q-n}=(0)$ if $q<n$ by Lemma 2.4 (which implies that the generator of rotations is a positive operator). So on $\mathscr{H}_{k}$ :

$$
B_{\alpha}=\sum_{m, n \geqq 0} e^{i(n-m-k) \alpha} \Gamma\left(e^{i q_{0}}\right) C_{m} D_{n} .
$$

This expression is not in fact an operator from $\mathscr{H}_{k}$ to $\mathscr{H}_{k+1}$, but note that when acting on $\mathscr{F}_{q}$ the sum over $n$ is actually finite. The sum over $m$ is made finite by letting $g: S^{1} \rightarrow \mathbb{C}$ be a trigonometric polynomial and setting

$$
B(g)=\frac{1}{\sqrt{2 \pi}} \int g(\alpha) B_{\alpha} d \alpha,
$$

or more precisely, writing $B_{\alpha, \lambda}$ for the expression on the right-hand side of (3.1) and noting that

$$
B(g) \xi=\lim _{\lambda \rightarrow 1} \frac{1}{\sqrt{2 \pi}} \int g(\alpha) B_{\alpha, \lambda} d \alpha \xi
$$

exists for all $\xi$ in the subspace of $\mathscr{H}_{k}$ consisting of finite linear combinations of eigenvectors of the rotation group. In fact this subspace is invariant under the action of $B(g)$. This observation means that the manipulations in Lemma 3.1 below are easily justified. Before stating this result note that the adjoint $B_{\alpha}^{*}$ of $B_{\alpha}$ is defined via

and

$$
\begin{aligned}
B_{\alpha}^{*} & =-i \lim _{\lambda \rightarrow 1} \Gamma\left(e^{-i q-\alpha}\right) \exp -i \beta\left(f_{\alpha, \lambda}\right)^{*} \exp -i \beta\left(f_{\alpha, \lambda}\right) \\
& =\lim _{\lambda \rightarrow 1} B_{\alpha, \lambda}^{*}
\end{aligned}
$$

$$
B^{*}(g)=\frac{1}{\sqrt{2 \pi}} \int \overline{g(\alpha)} B_{\alpha}^{*} d \alpha .
$$

We remark that the factor $e^{i \alpha}$ in $B_{\alpha}$ is replaced by $-i$ in $B_{\alpha}^{*}$ because

On $\mathscr{H}_{k}, B_{\alpha}^{*}$ has the form

$$
\Gamma\left(e^{i q-\alpha}\right)^{-1}=-i e^{i \alpha} \Gamma\left(e^{i q-\alpha}\right) .
$$

$$
B_{\alpha}^{*}=\sum_{m, n \geqq 0} e^{-i(m-n-k+1) \alpha} \Gamma\left(e^{i q_{0}}\right)^{-1} \tilde{C}_{m} \tilde{D}_{n},
$$

where $\tilde{C}_{m}, \tilde{D}_{n}$ are defined similarly to $C_{m}$ and $D_{n}$, and take $\mathscr{F}_{q}$ to $\mathscr{F}_{q+m}$ and $\mathscr{F}_{q}$ to $\mathscr{F}_{q-n}$ respectively.

Now we have

Lemma 3.1. For $f \in \mathscr{V}$ and $g, h \in L^{2}\left(S^{1}, \mathbb{C}\right)$, we have

(i) $[J(f), B(g)]_{-}=B(f g)$;

(ii) $\left[J(f), B(g)^{*}\right]_{-}=-B(f g)^{*}$;

(iii) $\left[B(g), B(h)^{*}\right]_{+}=\langle h, g\rangle_{L^{2}}$;

(iv) $[B(g), B(h)]_{+}=0=\left[B(g)^{*}, B(h)^{*}\right]_{+}$.

Proof. We follow Proposition 4.2 of [4].

(i) $\left[J(f), B_{\alpha}\right]_{-}=\lim _{\lambda \rightarrow 1}\left[J(f), B_{\alpha, \lambda}\right]_{-}$and $B_{\alpha, \lambda}$ differs from $\Gamma\left(\gamma_{\alpha, \lambda}\right)$ by a constant so that using the definition of $\tilde{\sigma}$ there is a function $f_{\lambda}(\alpha)$ with

$$
\left[J(f), \Gamma\left(\gamma_{\alpha, \lambda}\right)\right]_{-}=f_{\lambda}(\alpha) \Gamma\left(\gamma_{\alpha, \lambda}\right)
$$

and $f_{\lambda}(\alpha) \rightarrow f(\alpha)$ as $\lambda \rightarrow 1$. Hence (i) follows with (ii) similarly. 

obtain

(iii) We calculate $\left[B_{\alpha}, B_{\alpha^{\prime}}^{*}\right]_{+}=\lim _{\lambda \rightarrow 1}\left[B_{\alpha, \lambda}, B_{\alpha^{\prime}, \lambda}^{*}\right]_{+}$. On the right-hand side we

$$
\begin{aligned}
& \lim _{\lambda \rightarrow 1} \Gamma\left(e^{i\left(\alpha^{\prime}-\alpha\right)}\right) \exp \left[i [ \beta ( f _ { \alpha , \lambda } ) ^ { * } - \beta ( f _ { \alpha ^ { \prime } , \lambda } ) ^ { * } ] \operatorname { e x p } \left[i\left[\beta\left(f_{\alpha^{\prime}, \lambda}\right)-\beta\left(f_{\alpha^{\prime}, \lambda}\right)\right]\right.\right. \\
& \quad\left[\frac{e^{i\left(\alpha-\alpha^{\prime}\right)}}{1-\lambda^{2} e^{i\left(\alpha-\alpha^{\prime}\right)}}+\frac{1}{1-\lambda^{2} e^{-i\left(\alpha-\alpha^{\prime}\right)}}\right] .
\end{aligned}
$$

The factor in brackets converges to $2 \pi \delta(\alpha-\alpha)$.

(iv) A calculation similar to (iii) gives the analogous factor in brackets as

$$
-i e^{i \alpha^{\prime}}\left(1-\lambda^{2} e^{i\left(\alpha-\alpha^{\prime}\right)}\right)-i e^{i \alpha}\left(1-\lambda^{2} e^{-i\left(\alpha-\alpha^{\prime}\right)}\right),
$$

which converges to zero as $\lambda \rightarrow 1$.

Note that the anticommutation relations in (iii) and (iv) allow us to extend from trignometric polynomials to arbitrary elements of $L^{2}\left(S^{1}, \mathbb{C}\right)$ as smearing functions for the $B_{\alpha}$.

In [4] the corresponding limiting operators $B(g)$ were found to satisfy the commutation relations for a projective representation of $\operatorname{Map}\left(S^{1}, \operatorname{su}(2)\right)$ $(\operatorname{su}(2)=$ Lie algebra of $S U(2))$, the Lie algebra of $\operatorname{Map}\left(S^{1}, S U(2)\right)$. However we have "fermion" operators due to the different cocycle on $\mathscr{M}$.

Lemma 3.2. If $g_{j}=0, j \geqq 0$, then $B(g) \Omega=0$, while if $g_{j}=0, j<0$, then $B(g)^{*} \Omega=0$.

Proof. Using the definition of $B(g)$ and the fact that $\Omega$ is in the zero eigenspace of the generator of rotations, we have

$$
B(g) \Omega=\sum_{n>m \geqq 0} g_{m-n} \Gamma\left(e^{i q_{0}}\right) C_{m} D_{n} \Omega
$$

whenever $g_{j}=0, j \geqq 0$. But then $D_{n} \Omega=0$ for every $n$ in the sum, and so $B(g) \Omega=0$. The argument for $B(g)^{*}$ is identical.

From the preceding lemma one may compute

$$
\left\langle\Omega, B(g) B(h)^{*} \Omega\right\rangle=\left\langle h, P_{-} g\right\rangle,
$$

and by a standard argument also compute that the state, on the CAR algebra generated by $\left\{B(g), B(g)^{*} \mid g \in L^{2}\left(S^{1}, \mathbb{C}\right)\right\}$, determined by the cyclic vector $\Omega$, is exactly the state $\omega_{P_{-}}$of the introduction. In other words the representation of the CAR afforded by these operators is equivalent to the original one, but in fact we have more:

Proposition 3.3. For each $g \in L^{2}\left(S^{1}, \mathbb{C}\right), \psi(g)^{*}=B(g)$.

Proof. We will show that the matrix elements of $\psi(g)^{*}$ and $B(g)$ between states of the form

$$
J\left(f_{1}\right) \ldots J\left(f_{n}\right) \Gamma(V)^{m} \Omega
$$

are equal. By Theorem 2.3 such states form a total set in $\mathscr{F}$, and hence this will prove that $\psi(g)^{*}$ and $B(g)$ are equal. We break the proof into two assertions.

We claim that for each $\phi \in \operatorname{Map}\left(S^{1}, \mathbb{T}\right)$ and $g \in L^{2}\left(S^{1}, \mathbb{C}\right)$,

$$
\bar{\Gamma}(\phi) B(g) \Gamma(\phi)^{-1}=B(\phi g) \text {. }
$$

To see this note that if $\phi$ has winding number $n$ then $\phi V^{-n}$ has winding number zero and so lies in $\mathscr{M}_{0}$. Now Lemma 3.1, together with a simple calculation 
in the case of the constant functions in $\mathscr{M}_{0}$, shows that $\Gamma\left(\phi V^{-n}\right) B(g) \Gamma\left(\phi V^{-n}\right)^{-1}=B\left(\phi V^{-n} g\right)$. Thus (3.3) will follow provided we can show $\Gamma(V) B(g) \Gamma(V)^{-1}=B\left(e^{i v} g\right)$. But this last relation is a consequence of $\Gamma(V) B_{\alpha, \lambda} \Gamma(V)^{-1}=e^{i \alpha} B_{\alpha, \lambda}$. As $B_{\alpha, \lambda}$ differs from $\Gamma\left(e^{i \tilde{f}_{\alpha, \lambda}}\right)$ by a scalar this requires only that we prove $\exp -i \tilde{\sigma}\left(v, \tilde{f}_{\alpha, \lambda}\right)=\exp i \alpha$, which is true by a simple calculation using the definition of $\tilde{\sigma}$.

Now by (3.3), in calculating matrix elements between states of the form (3.2) we may move $B(g)$ through until it hits the vacuum $\Omega$. Consequently if we show $B(g) \Omega=\psi(g)^{*} \Omega$ for $g \in L^{2}\left(S^{1}, \mathbb{C}\right)$ then we are through.

Now to prove that the matrix element of $B(g)$ :

$$
M=\left\langle J\left(f_{1}\right) \ldots J\left(f_{n}\right) \Gamma(V)^{m} \Omega, B(g) \Omega\right\rangle
$$

is equal to the corresponding matrix element of $\psi(g)^{*}$ we use induction on $n$.

As $\Gamma(V)$ and $J\left(f_{j}\right)$ commute, we have $M=\left\langle\Gamma(V)^{m} \beta\left(f_{1}\right)^{*} \ldots \beta\left(f_{n}\right)^{*} \Omega, B(g) \Omega\right\rangle=\left\langle\beta\left(f_{2}\right)^{*} \ldots \beta\left(f_{n}\right)^{*} \Gamma(V)^{m} \Omega,\left[\beta\left(f_{1}\right), B(g)\right] \Omega\right\rangle$, as $\beta\left(f_{1}\right) \Omega=0$. Hence, using the relations in Lemma 3.1, we conclude that induction on $n$ establishes the result once we have shown

$$
\left\langle\Gamma(V)^{m} \Omega, B(g) \Omega\right\rangle=\left\langle\Gamma(V)^{m} \Omega, \psi(g)^{*} \Omega\right\rangle .
$$

But from Theorem 2.3 both sides of this relation are zero unless $m=1$. Now

$$
\langle\Gamma(V) \Omega, B(g) \Omega\rangle=\lim _{\lambda \rightarrow 1} \frac{1}{\sqrt{2 \pi}} \int_{0}^{2 \pi} \operatorname{dag}(\alpha)\left\langle\Gamma(V) \Omega, \Gamma\left(\gamma_{\alpha, \lambda}\right) \Omega\right\rangle c_{\alpha, \lambda},
$$

where

$$
c_{\alpha, \lambda}=\exp \left[-\frac{i}{2}\left(f_{\alpha, \lambda}^{+}(0)+f_{\alpha, \lambda}^{-}(0)\right)+i \alpha\right] \cdot\left(1-\lambda^{2}\right)^{-1 / 2} .
$$

But

$$
\begin{aligned}
\left\langle\Gamma(V) \Omega, \Gamma\left(\gamma_{\alpha, \lambda}\right) \Omega\right\rangle & =i\left\langle\Omega, \Gamma\left(e^{i\left(f_{\alpha, \gamma}-v\right)}\right) \Omega\right\rangle \exp i \sigma\left(v, f_{\alpha, \lambda}\right) \\
& =\left(1-\lambda^{2}\right)^{1 / 2} \exp \left[-i \alpha+\frac{i}{2}\left(f_{\alpha, \lambda}^{+}(0)+f_{\alpha, \lambda}^{-}(0)\right)\right]
\end{aligned}
$$

Thus

$$
\langle\Gamma(V) \Omega, B(g) \Omega\rangle=\frac{1}{\sqrt{2 \pi}} \int_{0}^{2 \pi} g(\alpha) d \alpha
$$

On the other hand

$$
\Gamma(V) \Omega=\psi^{*}\left(e_{0}\right) \Omega
$$

so that

$$
\left\langle\Gamma(V) \Omega, \psi^{*}(g) \Omega\right\rangle=\left\langle e_{0}, g\right\rangle=\frac{1}{\sqrt{2 \pi}} \int_{0}^{2 \pi} d \alpha g(\alpha),
$$

completing the proof. 
Remark 3.4. We thank the referee for help with the preceding proof.

As is noted in [4], a double cover of Diff $S^{1}$ acts on the central extension of $\mathscr{M}$ determined by $e^{i \sigma}$. Just as in [4] one obtains therefore a representation of Diff $S^{1}$ on $\mathscr{F}$ which leaves each $\mathscr{H}_{k}$ invariant. It is not difficult to see that, as Diff $S^{1}$ embeds in the symplectic group of $\bar{V}$ in exactly the same way as in [4], the representation of Diff $S^{1}$ on $H_{0}$ arises via the metaplectic representation of the symplectic group of $\overline{\mathscr{V}}$. It seems, however, that our analysis adds nothing new to the representation theory of Diff $S^{1}$.

One could also consider more general Bogoliubov transformations of $\mathscr{A}$, say those determined by the real orthogonal operators obtained by multiplication by smooth functions from $S^{1}$ to $O(2)$ on $L^{2}\left(S^{1}, \mathbb{C}\right)\left(\cong L^{2}\left(S^{1}, \mathbb{R}^{2}\right)\right.$ as a real Hilbert space). However these are only implemented when they take values in $\mathrm{SO}(2) \cong \mathrm{U}(1)$ and hence lie in $\mathscr{M}$.

Finally we note that this reconstruction of the fermions from the bosons is just exhibiting another model which "predicts its own superselection rules." See Streater [19] and references therein for this viewpoint.

Remark 3.5. Since this paper was first submitted new developments have taken place. With the exception of Proposition 3.3 the results of this paper were obtained independently and earlier by G. Segal and will appear in a forthcoming book on loop groups co-authored with A.N. Pressley. Work in progress by one of us (A.L. Carey) with S.N.M. Ruijsenaars has vastly extended the discussion in this paper.

\section{References}

1. Frenkel, I.B., Kac, V.G.: Invent. Math. 62, 23-66 (1980)

2. Frenkel, I B.: J. Funct Anal 44, 259-327 (1981)

3. Carey, A.L , Hurst, C.A., O'Brien, D.M.: Fermion currents in $1+1$ dimensions. J. Math. Phys. 24, 2212-2221 (1983)

4. Segal, G.: Unitary representations of some infinite dimensional groups. Commun. Math. Phys. 80, 301-362 (1981)

5. Dell'Antonio, G.F., Frishman, Y., Zwanziger, D.: Thirring model in terms of currents: Solution and light-one expansions. Phys. Rev. D 6, 988-1007 (1972)

6. Coleman, S.: Quantum sine-Gordon equation as a massive Thirring model. Phys. Rev. D 11, 2088-2097 (1975)

7. Ringwood, G.A.: The fermion-boson correspondence is a Klein transformation. Nuovo Cimento 54A, 483-494 (1979)

8 Ruijsenaars, S.N.M.: Integrable quantum field theories and Bogoliubov transformations. Ann Phys. 132, 328-382 (1981)

9. Heindenreich, R, Seiler, R., Uhlenbrock, D A : The Luttinger model. J Stat. Phys. 22, 27 (1980)

10. Carey, A L, Hurst, C.A., O’Brien, D.M.: J. Funct. Anal. 48, 360-393 (1982)

11. Carey, A.L, O’Brien, D.M.: Absence of vacuum polarisation in Fock space Lett. Math. Phys. 6, 335-340 (1982)

12. Douglas, R.G.: Banach algebra techniques in operator theory New York: Academic Press 1972

13. Baggett, L., Kleppner, A.: J. Funct Anal. 14, 299-324 (1978)

14. Hannabuss, K.C.: J Funct. Anal. 34, 144-165 (1979)

15. Kleppner, A.: Math Ann. 158, 11-34 (1965) 
16. Lundberg, L.-E.:Quasi-free "second quantization". Commun. Math.Phys. 50, 103-112 (1976) The calculations immediately preceding Theorem 2.3 may also be found in the unpublished paper of L.E. Lundberg: Observable algebra approach to the Thirring-Schwinger model

17. Ruijsenaars, S.N.M.: On Bogoliubov transformations for systems of relativistic charged particles. J. Math. Phys. 18, 517-526 (1977)

18. Uhlenbrock, D.A.: Fermions and associated bosons of one-dimensional model. Commun. Math. Phys. 4, 94 (1967)

19. Streater, R.F.: In: Physical reality and mathematical description. Enz, C.P., Mehra, J. (eds.). Boston: Reidel 1974

Communicated by H. Araki

Received May 19, 1982; in revised form December 3, 1984 